\title{
Comparison of Multivariate Curve Resolution Strategies in Quantitative LCxLC: Application to the Quantification of Furanocoumarins in Apiaceous Vegetables
}

\author{
Daniel W. Cook ${ }^{1}$, Mackenzie L. Burnham², David C. Harmes ${ }^{2}$, Dwight R. Stoll ${ }^{2}$, Sarah C. Rutan ${ }^{1}$
}

\section{Abstract}

Comprehensive two-dimensional liquid chromatography $(\mathrm{LC} \times \mathrm{LC})$ has been gaining popularity for the analysis of complex samples in a wide range of fields including metabolomics, environmental analysis, and food analysis. While LCXLC can provide greater chromatographic resolution than onedimensional LC (1D-LC), overlapping peaks are often still present in separations of complex samples, a problem that can be alleviated by chemometric curve resolution techniques such as multivariate curve resolution-alternating least squares (MCR-ALS). MCR-ALS has also been previously shown to assist in the quantitative analysis of LC x LC data by isolating pure analyte signals from background signals which are often present at higher levels in LC x LC compared to 1D-LC. In this work we present the analysis of a dataset from the LCXLC analyses of parsley, parsnip and celery samples for the presence and concentrations of 14 furanocoumarins. Several MCR-ALS implementations are compared for the analysis of LCXLC data. These implementations include analyzing the LCx LC chromatogram alone, analyzing the one-dimensional chromatogram alone, as well as two hybrid approaches that make use of both the first and second dimension chromatograms. Furthermore, we compared manual integration of resolved chromatograms versus a simple summation approach, using the resolved chromatographic peaks in both cases. It is found that manual integration of the resolved $L C \times L C$ chromatograms provides the best quantification as measured by the consistency between replicate injections. If the summation approach is desired for automation, the choice of MCR-ALS implementation has a large effect on the precision of the analysis. Based on these results, the concentrations of the 14 furanocoumarins are 
determined in the three apiaceous vegetable types by analyzing the LCXLC chromatograms with MCRALS and manual integration for peak area determination. The concentrations of the analytes are found to vary greatly between samples, even within a single vegetable type. 


\section{Introduction}

To better understand how diet plays a role in our health, researchers must be able to quantify naturally occurring compounds in food to enable further studies of the effects, positive or negative, of consuming these foods. One particular class of compounds of interest is furanocoumarins, which are found in many plant varieties including citrus, such as lemons and grapefruit $[1,2]$, and apiaceous vegetables, such as parsley, parsnips, and celery $[3,4]$. These compounds have long been known to be biologically active, affecting several systems in the body, particularly the P450 enzymes. P450 enzymes are found primarily in the liver and play a crucial role in the metabolism of many medicines and endogenous compounds. The well-known "grapefruit juice effect," in which consuming grapefruit juice can alter the metabolism of many drugs, is caused mainly by the inhibition of CYP3A4 by furanocoumarin compounds that are present in grapefruit juice [2]. It has also been suggested that inhibition of P450 enzymes, particularly of CYP1A2, may suppress procarcinogen activation [4]. Furanocoumarins are also known to cause photosensitivity of the skin, a property which can be harnessed to enhance ultraviolet treatments for skin diseases such as psoriasis and vitiligo $[5,6]$. In order to further understand how dietary exposure to these compounds affects a person's health, it is important to be able to detect and quantify the levels at which these compounds are present in different foods.

One promising approach for the analysis for complex mixtures such as those found in food analysis is the use of comprehensive two-dimensional liquid chromatography (LC $\times$ LC) [7]. Utilizing two different chromatographic separations coupled together increases the separation space available, while also allowing for greater selectivity due to differences in retention mechanisms between the two columns. This allows for better separation of analytes, particularly in complex matrices such as those found in plant extracts; however, even with the improved separation, it is not uncommon for target 
analyte peaks to be overlapped with interfering compounds. Particularly in these cases, the choice of detector can greatly impact the results of the analysis. While the improved selectivity of mass spectrometric (MS) detection can facilitate separation of analyte signals, costs associated with both purchase and maintenance make it a relatively expensive choice for detection. Diode array detection (DAD), on the other hand is an inexpensive and robust detection method which often has superior quantitative characteristics in comparison to MS detection (i.e. linear range, precision, repeatability), making it a very attractive method for quantitative analyses. In general, however, the lower selectivity of DAD requires that analytes be separated at a chromatographic resolution $\left(R_{S}\right)$ of greater than one. Achieving this resolution typically requires increasing analysis time and varying experimental conditions until an optimal set of chromatographic conditions is found. This optimal set of conditions is not always easily found and may be impractical, particularly in LCXLC where two separation dimensions must be optimized while maintaining compatibility in terms of mobile phases and flow rates. Alternatively, rather than investing in an extensive search for the optimal chromatographic conditions to completely resolve analyte signals, chemometric data analysis methods can be used to detect and quantify analytes even in the presence of chromatographic overlap [8].

Curve resolution methods, such as multivariate curve resolution-alternating least squares (MCRALS) $[9,10]$, offer a useful complement to LCXLC analyses. MCR-ALS is a method that aims to mathematically resolve analyte signals from one another and from the background signals. Analysis of LCXLC data using MCR-ALS results in a resolved two-dimensional (2D) chromatogram and a resolved spectrum for each analyte. With these resolved chromatograms, quantitative analysis can be performed. Typically, peaks are integrated to obtain the area under the peak, either manually or with peak detection software; however, if background and noise are sufficiently reduced or eliminated, it is possible to simply integrate over the entire component elution window, simplifying the quantification process because the start and end points of the peak do not have to be identified. 
Here, we demonstrate the application of MCR-ALS to the analysis of data from LCXLC separations of apiaceous vegetable extracts, with a focus on quantification of 14 furanocoumarins in these extracts. MCR-ALS is performed on both the ${ }^{1} \mathrm{D}$ chromatograms, collected at the first dimension $\left({ }^{1} \mathrm{D}\right)$ detector, and the 2D chromatograms, in addition to our previously reported 2D assisted liquid chromatography (2DALC) approach [11]. In addition, two approaches to integration (manual and summation) are compared.

\section{Theory}

\subsection{Comprehensive Two-Dimensional Liquid Chromatography (LCXLC)}

$\mathrm{LC} \times \mathrm{LC}$ couples two separations, with different separation chemistries, in order to increase the available separation space and overall selectivity of the method for analytes contained in the mixture. The principles of $L C \times L C$ have been described extensively elsewhere [12-14]. Briefly, the ${ }^{1} D$ separation is performed similarly to traditional $1 \mathrm{D}-\mathrm{LC}$. The effluent from the ${ }^{1} \mathrm{D}$ column is collected in a sampling device, usually a valve or series of valves. These samples are then passed sequentially to the ${ }^{2} \mathrm{D}$ column and then to a detector, often a diode array detector (DAD) or mass spectrometer (MS). The detector produces a series of ${ }^{2} D$ chromatograms, one for each ${ }^{1} D$ effluent fraction, which then must be "folded" into an LCXLC chromatogram [12]. In the current work, a detector was also placed after the ${ }^{1} \mathrm{D}$ column, before the sampling valve, in order to collect a ${ }^{1} \mathrm{D}$ chromatogram. This allows for the comparison of the analyses of the $L C \times L C$ chromatogram and the ${ }^{1} D$ chromatogram before sampling, particularly in terms of quantitative performance.

\subsection{Multivariate Curve Resolution-Alternating Least Squares (MCR-ALS)}

Multivariate curve resolution-alternating least squares (MCR-ALS) is a method to mathematically resolve pure analyte signals from one another and from background contributions to the signal $[9,10]$. 
The bilinear model is shown in Eq. (1), where $\mathbf{X}$ is the raw second-order data (i.e., a spectrochromatogram) and $\mathbf{C}$ and $\mathbf{S}$ are the chromatographic and spectral profiles, respectively, when chromatographic data are analyzed. The superscript $T$ represents a transpose of the matrix. $\mathbf{E}$ is the residual error from fitting the model.

$$
X=\text { CS }^{\top}+E
$$

This equation is solved via alternating least squares (ALS) in which an initial guess is used to initiate the optimization of $\mathbf{C}$ and $\mathbf{S}$. Through appropriate use of constraints during the ALS process (described below), the resulting $\mathbf{C}$ and $\mathbf{S}$ matrices correspond to true chemical species resolved into separate components, represented by vectors within these matrices.

The initial guess used to initiate ALS can be estimates of the chromatographic profiles, $\mathbf{C}$, but most often are estimates of the spectral profiles, $\mathbf{S}$. Multiple methods have been reported to obtain initial guesses for MCR-ALS [15-17]. In this work we use the iterative orthogonal projection approach (IOPA) [11]. IOPA aims to extract spectra from the raw data that are most orthogonal, or most dissimilar, from one another. The number of spectra that are extracted is defined by the user prior to initiating IOPA based on knowledge of the sample or by using scree plots [18]. This initial guess is then used to initiate ALS. The number of spectra extracted for the initial guess defines the number of components used to model the data during MCR-ALS.

\subsubsection{Data Structure}

As shown in Eq. (1), MCR-ALS decomposes a matrix, $\mathbf{X}$, into two separate matrices, $\mathbf{C}$ and $\mathbf{S}$. When a single 1D-LC-DAD analysis is performed, a single data matrix is formed with time along one dimension and wavelength along the second dimension of the $\mathbf{X}$ matrix. This matrix can be analyzed without further data rearrangement; however, it is often desired to analyze multiple samples 
simultaneously, expanding the raw data to form a third-order data cube, which must be rearranged to a second-order data matrix for analysis by MCR-ALS. This rearrangement is accomplished by concatenating all samples along a single time axis, keeping the spectral axis intact [19]. This concatenation can also be used to rearrange LCXLC-DAD for MCR-ALS analysis, which forms a fourthorder data array when multiple samples are analyzed. First, all ${ }^{2} \mathrm{D}$ chromatograms are concatenated along a single time dimension. This process is often referred to as 'unfolding' the chromatogram and is typically performed whenever LCxLC chromatograms are analyzed. These unfolded LCxLC chromatograms are then concatenated again along the time dimension, giving a single augmented time dimension while still retaining the spectral dimension. This process is graphically depicted in Fig. 1.

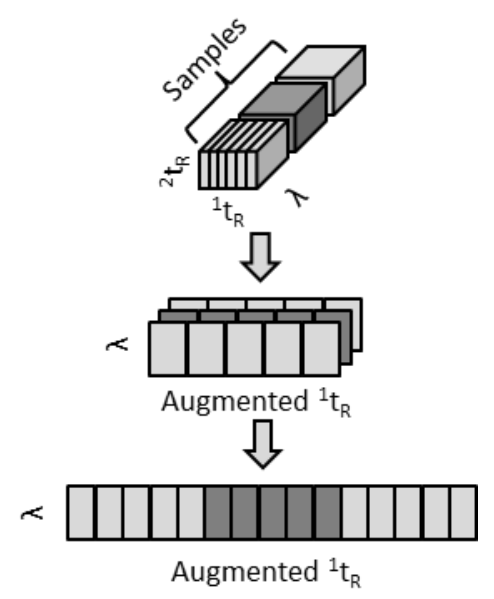

Figure 1. Graphical representation of the data rearrangement process for reshaping a fourth-order data array obtained from a multi-sample LCXLC-DAD analysis consisting of two time dimensions $\left({ }^{1} t_{R}\right.$ and $\left.{ }^{2} t_{R}\right)$, a spectral dimension $(\lambda)$, and a sample dimension. The fourth-order array is first unfolded along the time axes and finally multiple samples are concatenated along the new time axis.

\subsubsection{Constraints}

MCR-ALS analysis relies on the application of constraints during the ALS procedure. These constraints force the chromatographic and spectral profiles to have chemically sensible shapes and 
characteristics. Without constraints, Eq. (1) has an infinite number of mathematically valid solutions, a problem known as rotational ambiguity [20]. With constraints, the range of possible solutions is greatly reduced. When these constraints are correctly chosen, the results of MCR-ALS represent the true, underlying analyte signals. The most common of these constraints is nonnegativity, in which intensities of components corresponding to chemical species are forced to be greater than or equal to zero, based on the realization that concentrations cannot be negative. Other constraints commonly employed are: unimodality, forcing a single signal maximum per component (relevant for the chromatographic profiles); selectivity, forcing certain regions in the chromatographic or spectral profiles to known values; and normalization, which prevents intensity ambiguities and allows the correlation between peak area or intensity, and concentration [19]. These constraints are applied to each component individually and to each dimension independently, resulting in a very flexible, but powerful method.

\subsection{D Assisted Liquid Chromatography (2DALC)}

Recently, we reported a strategy for analyzing LCXLC data that we termed 2D Assisted Liquid Chromatography (2DALC) [11]. After analyzing LCXLC-DAD data with MCR-ALS, the resolved analyte spectra are used as the initial guess for a second analysis with MCR-ALS, this time on the ${ }^{1} D$ chromatogram, obtained from a detector placed at the end of the ${ }^{1} \mathrm{D}$ column. The resolved ${ }^{1} \mathrm{D}$ chromatograms are then used for quantification. This allows for the chromatographic resolution of $L C \times L C$ to be combined with the superior quantitative characteristics of $1 D-L C$, which result from the higher $\mathrm{S} / \mathrm{N}$ of the ${ }^{1} \mathrm{D}$ detector data. In the original paper [11], it was found that 2DALC is a useful tool in

situations of severe ${ }^{1} \mathrm{D}$ peak overlap, high ${ }^{2} \mathrm{D}$ background, and/or highly similar analyte spectra. 2DALC was used in the current work for comparison of quantitative performance with ${ }^{1} D$ and $2 D$ MCR-ALS analysis for the current dataset.

\subsubsection{Combined 2DALC (c2DALC)}


In the present work, we have modified the 2DALC algorithm described above to carry out the analysis of the ${ }^{1} \mathrm{D}$ and $2 \mathrm{D}$ data in a single step, and have termed this method combined 2DALC (c2DALC). This method takes advantage of the concatenation process discussed in a section 2.2.1, above. After the rearrangement of the $2 \mathrm{D}$ chromatogram to concatenated ${ }^{2} \mathrm{D}$ chromatograms, the ${ }^{1} \mathrm{D}$ chromatogram is concatenated to this reshaped 2D chromatogram along the same augmented axis, a process depicted graphically in Fig. 2 . This rearrangement is possible because both the ${ }^{1} \mathrm{D}$ and ${ }^{2} \mathrm{D}$ detectors collect spectra using the same wavelength range and at the same spectral resolution, meaning that spectra from both detectors have identical wavelength axes $[19,21]$. By concatenating the ${ }^{1} D$ and ${ }^{2} D$ chromatograms into a single rearranged chromatogram and performing a single MCR-ALS analysis, as opposed to a two-step process in 2DALC, the spectral information found in the 2D chromatogram can more directly assist in the resolution of the ${ }^{1} D$ chromatogram, assuming the spectral responses are the same between the two detectors. As in 2DALC, the resolved ${ }^{1} \mathrm{D}$ chromatogram is used for peak integration and subsequent quantification.

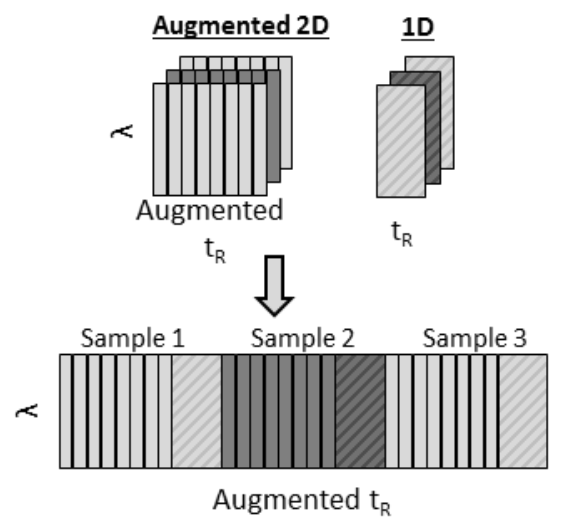

Figure 2. Graphical representation of c2DALC data rearrangement. The reshaped 2D chromatogram is first concatenated with the ${ }^{1} \mathrm{D}$ chromatogram. Then, the samples are concatenated into a single matrix.

\section{Experimental}


listed in Fig. 3, along with one internal standard, 4-chlorobenzophenone (CBP). Samples of three apiaceous vegetable types - parsley, parsnips, and celery - were then analyzed for the presence and concentrations of these 14 target compounds.

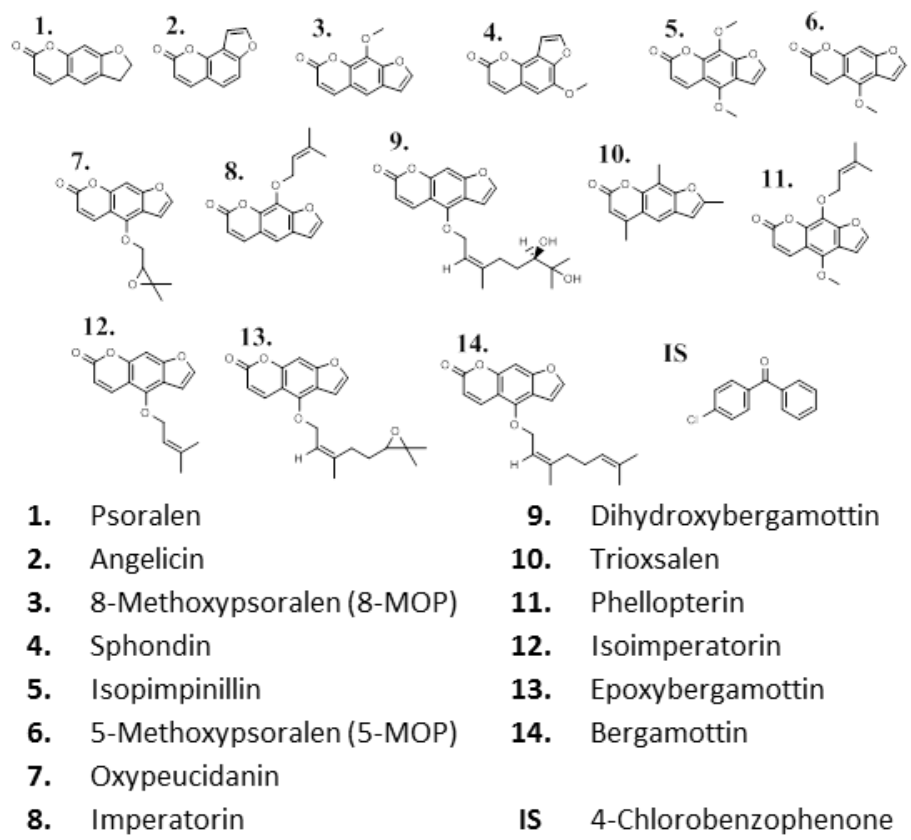

Figure 3. Chemical structures of the 14 furanocoumarins targeted in the current work along with the internal standard (IS) used.

\subsection{Materials}

The following materials were received from Sigma Aldrich (St. Louis, MO): acetonitrile (HPLC grade $\geq 99.9 \%$ ), acetic acid (reagent grade $\geq 99.0 \%$ ), sodium phosphate (monobasic monohydrate) (ACS reagent $\geq 98.0 \%$ ), sodium phosphate (dibasic) (ACS reagent $\geq 99 \%$ ), sodium acetate (ReagentPlus $\geq 99 \%$ ), methanol (Sigma Aldrich; HPLC grade), psoralen, angelicin, 8-MOP, 5-MOP, and trioxsalen. Phosphoric acid (HPLC grade 85\%) was obtained from Fisher Scientific (Fairlawn, NJ). Magnesium sulfate was obtained from J.T. Baker (Phillipsburg, NJ). Sphondin, bergamottin, 6,7-dihydroxybergamottin, 
epoxybergamottin, isoimperatorin, oxypeucedanin, and phellopterin were obtained from Herboreal Ltd. (Edinburgh, UK). Isopimpinellin and imperatorin were obtained from Indofine Chemical Company (Hillsborough, NJ), while the internal standard 4-chlorobenzophenone (CBP) was obtained from Matheson Coleman \& Bell (Cincinnati, OH). HPLC-grade water was prepared in-house by use of a Milli-Q Nanopure water purification system (Billerica, MA) with a pyrogen-, DNase-, and RNase-free final cartridge.

\subsection{Plant Extracts}

Organically grown vegetables were purchased at several different time points from a local market (St. Paul, MN). Samples were prepared combining $5.0 \mathrm{~g}$ of wet vegetable matter with $10 \mathrm{~mL}$ of water and $10 \mathrm{~mL}$ of acetonitrile (ACN) containing $0.1 \%$ acetic acid. This mixture was then homogenized in an Ultra Turrax T25 Homogenizer (IKA Laboratories, Wilmington, NC) for 3 min. A 1 g portion of sodium acetate and $4 \mathrm{~g}$ of magnesium sulfate were added to the homogenate and hand-mixed by inversion for $2 \mathrm{~min}$. Samples were then centrifuged at 3,000 rpm for $5 \mathrm{~min}$. at room temperature. Following centrifugation, $1.8 \mathrm{~mL}$ of the supernatant was transferred to a $2-\mathrm{mL}$ dispersive solid phase extraction (dSPE) tube (United Science 20 CarbonX QuEChERS; Center City, MN; part number 05040114). Water was added to a total volume of $2 \mathrm{~mL}$ (final composition of $10 \%$ water: $90 \% \mathrm{ACN}$ ) and tubes were vortexed with a Mini Vortex (VWR Part Number 12620-852, Radnor, PA) at the maximum speed setting. Samples were again centrifuged at $6,000 \mathrm{rpm}$ for $30 \mathrm{~s}$ and the supernatant was transferred to a HPLC vial and diluted 1:2 with water prior to LC analysis. Each extract was analyzed in duplicate via LCxLCDAD.

\subsection{Chromatographic Conditions}

The LCxLC analyses were performed on modules from the Agilent Technologies 1290 Infinity line (Agilent Technologies, Waldbronn, Germany). Samples were injected using an autosampler (Model 
G4226A). First and second dimension flows were generated using binary pumps (Model G4220A); a quaternary pump (Model DEQAT00023) was used to dilute ${ }^{1} \mathrm{D}$ effluent to focus analyte bands at the inlet of the ${ }^{2} \mathrm{D}$ column [22]. Thermostated column compartments were used in both dimensions (Model G1316C) and nominally identical diode-array UV detectors (DADs) (Model G4212A) were used at the outlets of the ${ }^{1} \mathrm{D}$ and ${ }^{2} \mathrm{D}$ columns. Low dispersion flow cells (800 $\mathrm{nL}$ volume, Model G4212-60038) were used in both the ${ }^{1} D$ and ${ }^{2} D$ DAD detectors. At the outlet of the ${ }^{1} D$ flow cell a pressure relief valve (Model G4212-60022) was used. A 2-position 8-port valve (Model 5067-4214) fitted with two $80 \mu \mathrm{L}$ stainlesssteel loops (Model 5067-5426) mounted on an external valve drive (Model G1170A) was used to transfer fractions of ${ }^{1} D$ effluent to the ${ }^{2} D$ column. The $2 D-L C$ instrument was controlled by OpenLab Chromatography Data System (Agilent Technologies, Rev. C.01.07[27]).

The ${ }^{1} \mathrm{D}$ separation was performed using an Agilent Poroshell 120 PFP (100 mm x $2.1 \mathrm{~mm}$ i.d.) column with $5 \mathrm{mF}$ sodium phosphate at $\mathrm{pH} 6(\mathrm{~A})$ and methanol (B). A gradient was used with the following conditions: $45-45-75-100-100-45-45 \%$ B from 0-12.5-29.5-31.5-33-33.01-36 min; this is shown in the supplemental Fig S.1. The temperature was held at $20^{\circ} \mathrm{C}$ and the flow rate was $0.25 \mathrm{~mL} / \mathrm{min}$ from 0-7.5 $\mathrm{min}$, and $0.125 \mathrm{~mL} / \mathrm{min}$ from 7.51 to $36 \mathrm{~min}$. Beginning at $7.0 \mathrm{~min}$ the ${ }^{1} \mathrm{D}$ effluent was diluted with $20 \mathrm{mM}$ phosphoric acid in water at $0.125 \mathrm{~mL} / \mathrm{min}$. The diluted effluent was collected in $80 \mu \mathrm{L}$ sample loops before injection into the ${ }^{2} \mathrm{D}$ column.

The second dimension $\left({ }^{2} D\right)$ separation was performed from 7.5-36 min using an Agilent Zorbax SB-C18 RRHD (50 mm x $3.0 \mathrm{~mm}$ i.d., $1.8 \mu \mathrm{m}$ ) column using $20 \mathrm{mM}$ phosphoric acid in water (A) and acetonitrile (B) as mobile phase components. The temperature was held at $50^{\circ} \mathrm{C}$ and the flow rate was $2.5 \mathrm{~mL} / \mathrm{min}$. The ${ }^{2} \mathrm{D}$ mobile phase was held isocratic at $42 \% \mathrm{~B}$ for the first $12.5 \mathrm{~min}$ and then a shifted gradient was employed as shown in supplemental Fig. S.1.

\subsection{Data Analysis}


All computations were performed on a Dell Precision T3600 with an Intel Xeon E5-1620 CPU at 3.60 GHz and 32.0 GB of RAM. Data files were converted from the Agilent .D format to MATLAB .mat format files using ACD/Lab Spectrus Processor (Advanced Chemical Development, Inc., Toronto, Canada). All other computational analyses were performed in MATLAB version R2013a (Mathworks, Inc., Natick, MA).

Prior to MCR-ALS analysis, the data were divided into sections to minimize chromatographic complexity and data size. The sections chosen for analysis are shown in Fig. 4. For MCR-ALS analysis, the full dataset was subdivided into three smaller datasets. Each dataset contained all samples from a single vegetable type $\left(n_{\text {parsley }}=56 ; n_{\text {parsnip }}=56 ; n_{\text {celery }}=54\right)$. Each dataset also contained two blanks (measured under identical chromatographic conditions) and three identical calibration sets (used to monitor drift over the course of the experimental data acquisition), each consisting of five standard mixtures with analyte concentrations ranging from $1-50 \mu \mathrm{g} / \mathrm{mL}$. Each standard mixture contained all target compounds at varying concentrations as listed in the supplemental Table S.1. Internal standard calibration curves were created by integrating the resolved chromatograms, with each of the methods described in the following section. Because the concentrations of many of the compounds were found at the low end of this concentration range, calibration standards that were much higher than the predicted concentration in the extract were eliminated from the calibration curves, and analyte concentrations were predicted with this new calibration curve. The narrower range of concentrations resulted in increased accuracy of prediction.

MCR-ALS was performed using an in-house algorithm which accepts LCXLC data in the original four-way data array $\left({ }^{2} D\right.$ time $x{ }^{1} D$ time $x$ spectra $x$ sample) or $1 D-L C$ data as a three-way array. The algorithm rearranges the data to the necessary two-way data structure, as described previously, and shown in Figs. 1 and 2. After each iteration the data are once again rearranged to the full four-way array 
to allow for the flexible implementation of constraints on the each of the dimensions -- ${ }^{1} D$ and ${ }^{2} D$ chromatograms, spectra, and samples -- of each component independently. The available constraints include non-negativity, unimodality, local rank in both the spectral and chromatographic dimensions, smoothness (based on Eilers' perfect smoother [23]), and trilinearity [24]. Especially important is the ability of our algorithm to constrain each ${ }^{2} \mathrm{D}$ chromatogram individually to be unimodal, allowing only a single peak per ${ }^{2} \mathrm{D}$ chromatogram. This was found to be particularly useful for reducing the contribution of residual background to the chemically relevant components (analytes). In the current work, nonnegativity and local rank were used in both the chromatographic and spectral dimensions. Local rank constraints allow the user to set regions in the chromatographic and/or spectral profiles to zerointensity. For spectral local rank, the knowledge that the target analytes do not absorb light at higher wavelengths was used to set wavelengths higher than $440 \mathrm{~nm}$ to zero intensity in the spectral profiles corresponding to the target analytes._Chromatographic local rank was implemented in regions where the chromatographic profiles of the target analytes contained no peak-like shapes as determined by visual inspection. A correspondence constraint was implemented as well, which informs the algorithm of blank runs in which the target analytes are not present and therefore sets those components to zerointensity across the entire chromatographic profiles for those analytes. Unimodality was used in some cases; however, in many cases, particularly when analyzing the ${ }^{1} \mathrm{D}$ chromatograms, interferents (as defined by compounds with identical spectra as our target analytes) were present in the resolved component chromatograms. This means that the true, underlying chromatographic profiles were not unimodal and implementing unimodality in these cases would incorrectly constrain the chromatographic profiles, leading to incorrect results. The decision whether to utilize a unimodality constraint was made on a case-by-case basis based on the presence of two (or more) peak-like shapes in the resolved chromatographic profile and the failure of MCR-ALS to adequately converge to a correct solution when 

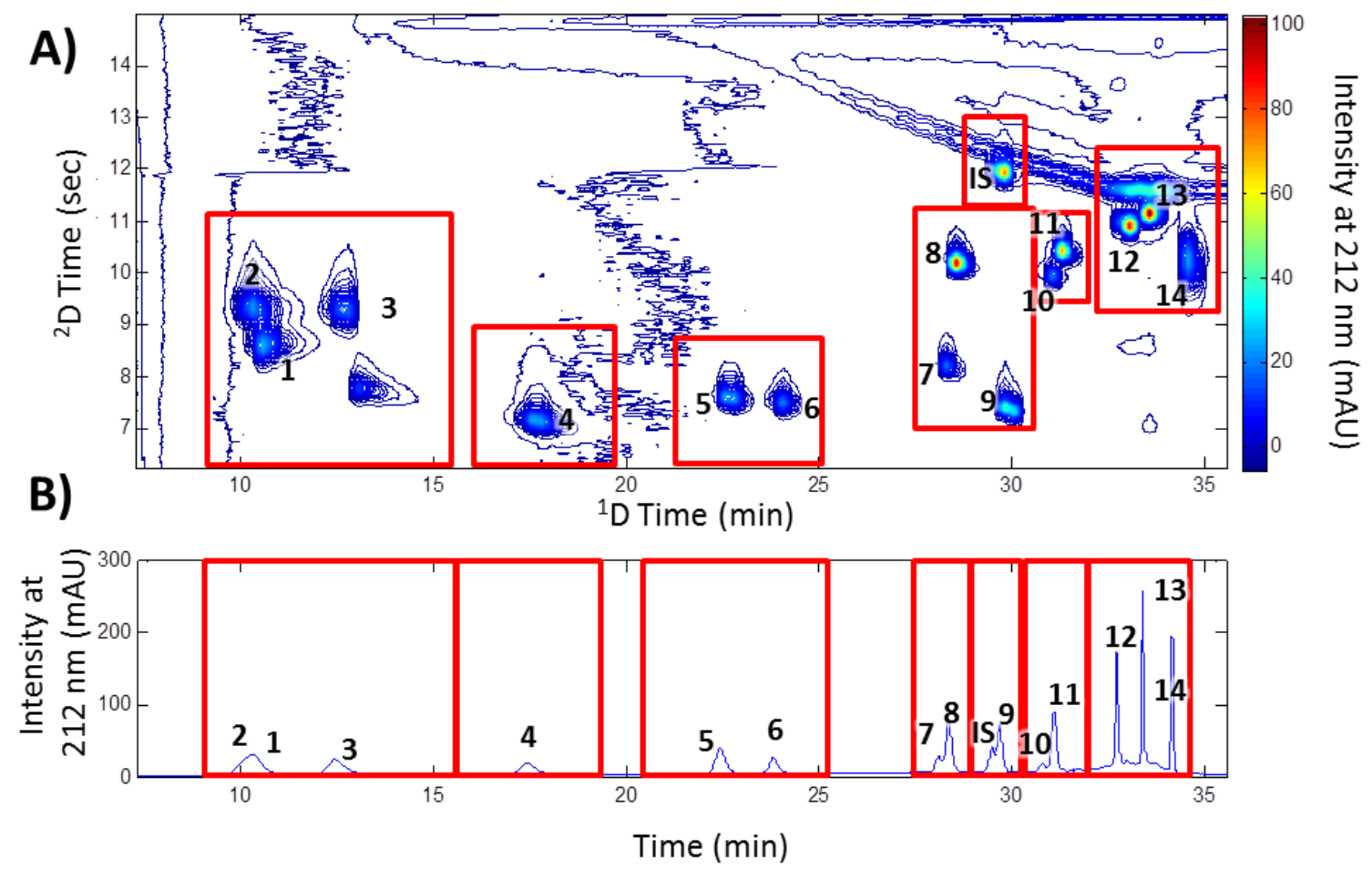

Figure 4. Representative chromatograms where all compounds concentrations are $10 \mu \mathrm{g} / \mathrm{mL}$. A and B show the ${ }^{1} \mathrm{D}$ and 2D chromatograms, respectively. The boxes indicate the windows chosen for analysis and the numbers labeling the peaks correspond to the compounds in Fig. 3. IS = internal standard.

MCR-ALS was performed on the data using four strategies: ${ }^{1} D, 2 D, 2 D A L C$, and $c 2 D A L C$. The ${ }^{1} D$ analysis corresponds to MCR-ALS being applied to only the ${ }^{1} \mathrm{D}$ chromatogram, collected at the ${ }^{1} \mathrm{D}$ detector. This analysis is representative of 1D-LC analysis without a second dimension of separation. The $2 \mathrm{D}$ analysis corresponds to MCR-ALS being applied to only the $2 \mathrm{D}$ chromatogram, collected at the ${ }^{2} \mathrm{D}$ detector. Finally, 2DALC and C2DALC are the strategies described in the Theory section, utilizing both the ${ }^{1} \mathrm{D}$ and $2 \mathrm{D}$ chromatograms. Two integration strategies were also compared. Manual integration involves 
the manual selection of peak boundaries, estimation of a linear background, and integration of the signal difference between the peak and the background. A simpler approach, termed summation in this work, involves the summation of all intensities of a resolved chromatographic peak across the entire analysis window. This approach assumes that any noise or background is sufficiently removed via MCRALS and that all signal intensity is due to the analyte.

\subsection{Simulated Data}

Because c2DALC assumes spectral profiles of each analyte remain constant between the ${ }^{1} \mathrm{D}$ and ${ }^{2} \mathrm{D}$ detectors, any shift due to solvent effects may affect its ability to resolve analyte signals. To investigate these effects, simulated data were created. Two datasets were simulated in an identical manner to our previous work [11]. Briefly, three Gaussian peaks were added onto real, independent LCxLC backgrounds to simulate three analyte chromatographic peaks. This was done for three 'samples' at three different 'concentrations.' The peak intensity for each analyte was varied independently across the samples to simulate different concentrations giving peak heights ranging from 100-600 mAU (at 244 $\mathrm{nm})$. Analyte spectra for each compound were obtained from the analysis of pure standards of each compound at both the ${ }^{1} \mathrm{D}$ and ${ }^{2} \mathrm{D}$ detector. Both detector signals were then subjected to MCR-ALS separately. This allowed for the recovery of pure analyte spectra which accurately reflected the differences between the two detectors. For the first dataset, spectra corresponding to three furanocoumarins -- psoralen, 8-MOP, and angelicin -- were used. For the second dataset, the compound spectra were taken from three amphetamine compounds: 3,4-methylenedioxy-methamphetamine (MDMA), methoxymethamphetamine (MoxyMeth), and methamphetamine (Mamp). For each of these sets of compounds data were simulated to mimic the type of spectral differences observed in the experimental spectra obtained from the ${ }^{1} D$ and ${ }^{2} D$ detectors in this study. The experimental spectra used for these simulations are shown in Fig. 5. In this figure, the differences in the spectral response 
between the ${ }^{1} D$ and ${ }^{2} D$ detectors are shown. The differences are slightly more pronounced in the furanocoumarin spectra than the amphetamine spectra. These two sets of analytes also display different spectral similarity between analytes where furanocoumarins have much more similar spectra (average correlation between analyte spectra: $\left.\overrightarrow{r^{2}}=0.96\right)$ than the amphetamines $\left(\overrightarrow{r^{2}}=0.46\right)$. In general, MCR-ALS performance improves with more dissimilar analyte spectra and we believe that the deleterious effect of the spectral differences is less pronounced when spectral similarity between the analyte spectra is low. MCR-ALS was performed with the four methods listed in the previous section and the resolved chromatographic profiles were quantified using the summation approach.
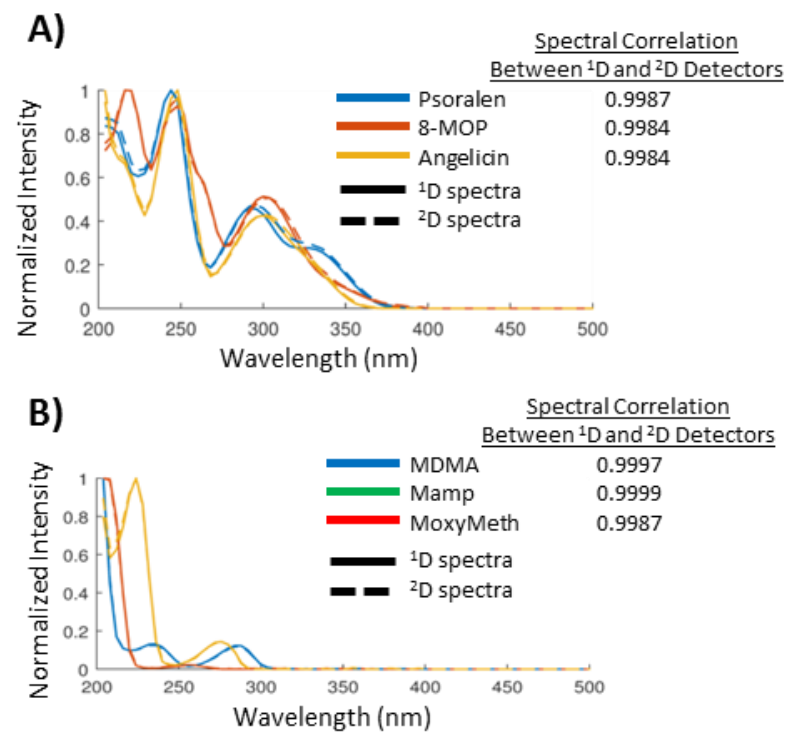

Figure 5. Spectra used for data simulation. (A) shows the furanocoumarin spectra and (B) represent the amphetamine spectra: methylenedioxymethamphetamine (MDMA), methoxymethamphetamine (MoxyMeth), and methamphetamine (Mamp). Spectral correlations $\left(r^{2}\right)$ are a measure of the spectral shifts between the ${ }^{1} \mathrm{D}$ spectra (solid lines) and the ${ }^{2} \mathrm{D}$ spectra (dashed lines). These correlation values were calculated over the spectral range from $200-400 \mathrm{~nm}$.

\section{Results}




\subsection{Simulated Data -- Combined 2DALC}

In order for C2DALC to be successful, the spectra of the individual compounds obtained at the ${ }^{1} D$ and ${ }^{2} \mathrm{D}$ detectors must be highly similar, due to the fact that all the data are combined into a single matrix. MCR-ALS requires that each analyte has a constant spectral profile that only varies in intensity. This property, known as bilinearity, is what allows MCR-ALS to resolve analyte peaks accurately. When the ${ }^{1} D$ and ${ }^{2} D$ spectra are slightly different the bilinearity assumption is incorrect and MCR-ALS fails. Figure 5 shows spectra obtained at both the ${ }^{1} D$ and ${ }^{2} D$ detectors. Slight spectral shifts between the data are observed, which we attribute to the different solvent polarities of the ${ }^{1} D$ and ${ }^{2} D$ mobile phases [25]. These experimental spectra were used to create simulated data for investigations into these solvent effects.

The results for the analysis of the simulated data using all four curve resolution techniques are shown in Fig. 6. The peak area precision was determined by first calculating the peak areas of the resolved chromatographic profiles. The peak areas for a given analyte were then normalized by the known 'concentration' in each sample, and the \%RSD of the resulting normalized areas was calculated. In general, as ${ }^{1} R_{S}$ increases, the performance of 1D-IOPA improved, as expected. This trend generally held for 2DALC and C2DALC, which both integrate the ${ }^{1} D$ chromatogram; however, the peak area precision was generally worse for c2DALC versus 2DALC. For these data, 2D-IOPA most often outperformed the other methods at low ${ }^{1} R_{S}$ values, with a few exceptions; this is most likely due to the additional chromatographic resolution of the compounds in the second dimension $\left({ }^{2} R_{S}=0.4\right)$ whereas MCR-ALS was less able to sufficiently resolve the ${ }^{1} D$ chromatograms at ${ }^{1} R_{s}<0.3$. The results for angelicin do not follow this trend. At ${ }^{1} R_{S}=0.2,{ }^{1} \mathrm{D}-$ IOPA is superior to the other methods. This is most likely due to the fact that the angelicin peak is overlapped on both sides with psoralen and 8-MOP in the ${ }^{2} \mathrm{D}$ 
separation. In the ${ }^{1} \mathrm{D}$ chromatogram, angelicin elutes last allowing for a greater portion of the peak not being contaminated by another peak and may explain the low \%RSD values for ${ }^{1} \mathrm{D}-\mathrm{IOPA}$.

Another interesting trend shown in Fig. 6 is that of c2DALC when the 2D portion of the resolved chromatogram is integrated (labeled as C2DALC (2D)), rather than integrating the ${ }^{1} \mathrm{D}$ portion (labeled as c2DALC). For many of the compounds, the \%RSD values are lowest for c2DALC (2D) at low ${ }^{1} R_{S}$ values and increase as ${ }^{1} R_{S}$ increases, correlating with that of 2D-IOPA. This is the opposite trend as c2DALC (integrating the ${ }^{1} \mathrm{D}$ portion of the resolved chromatogram), which follows a trend that is similar to the ${ }^{1} \mathrm{D}$-IOPA results. The goal of $\mathrm{C} 2 \mathrm{DALC}$ is the simultaneous optimization of the ${ }^{1} \mathrm{D}$ and $2 \mathrm{D}$ chromatographic (and spectral) profiles. Ideally, the optimal solution would match the true peak profiles for both the ${ }^{1} \mathrm{D}$ and 2D chromatograms (i.e., \%RSD minimized for both chromatograms); however, the opposing trends suggest that at low values of ${ }^{1} R_{S}$, c2DALC is optimizing the profiles based on the ${ }^{2} D$ spectra, which are more distinct due to improved resolution in the ${ }^{2} \mathrm{D}$ separation. At higher values of ${ }^{1} R_{S}$ C2DALC is optimizing based on the ${ }^{1} \mathrm{D}$ spectra, which are more distinct due to the higher $\mathrm{S} / \mathrm{N}$ in the ${ }^{1} \mathrm{D}$ separation. Due to this finding, and the observation that C2DALC did not provide a significant advantage over 2DALC, c2DALC was not used for analysis in further sections of this work. 
Figure 6. Comparison of peak area precision for the analysis of simulated data using each curve resolution implementation. Results from simulated data with two sets of analyte spectra are shown; furanocoumarins have much more similar spectra (average spectral correlation: $\overrightarrow{r^{2}}=0.96$ ) than amphetamines $\left(\overrightarrow{r^{2}}=0.46\right) .{ }^{2} R_{s}=0.4$. c2DALC (2D) represents the case in which c2DALC was performed and the 2D portion of the augmented data was integrated.

\subsection{Experimental Data -- Comparison of Integration Methods}

LCxLC-DAD analyses were carried out on samples from three apiaceous vegetable types parsley, parsnips, and celery. Using DADs placed at the end of the ${ }^{1} \mathrm{D}$ and ${ }^{2} \mathrm{D}$ columns, both ${ }^{1} \mathrm{D}$ and $2 \mathrm{D}$ chromatograms were collected, allowing for comparisons of quantitative performance for ${ }^{1} D-1 O P A, 2 D-$ IOPA, and 2DALC, as described in the Theory section. In addition to these methods, it is also crucial to investigate the impact of integration methods on quantitative performance.

Ideally, MCR-ALS completely resolves the pure analyte signals from the background and noise, leaving only signal intensity resulting from the analyte. In this case, peak areas could be calculated from a simple summation of all intensities from the resolved chromatographic profile from the beginning to 
A)

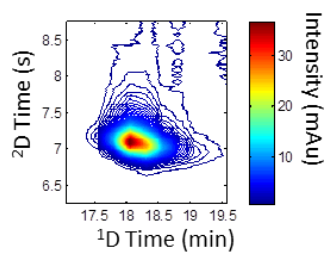

B)

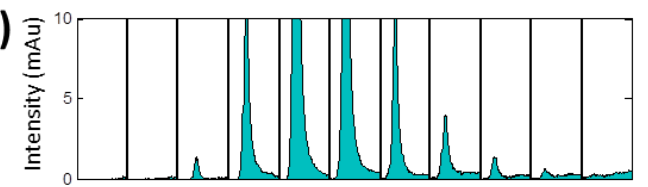

C)

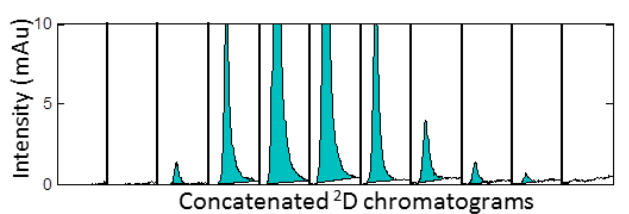

Figure 7. Graphical representation of integration methods compared. A shows a MCR-ALS resolved LCXLC chromatogram. B and $\mathbf{C}$ show the rearranged $\mathrm{LC} \times \mathrm{LC}$ chromatogram with simple summation and manual integration, respectively. The shading represents the area used for quantification. The percent difference in calculated peak area for simple summation and manual integration is $9.9 \%$. 

analyte concentrations of duplicate parsley samples (26 duplicate pairs) were summed and are shown in Fig. 8. This sum of differences metric allows for a comparison of the precision of manual integration and summation among the three analysis methods tested $-{ }^{1} \mathrm{D}, 2 \mathrm{D}$, and $2 \mathrm{DALC}$. By comparing the solid and patterned bars in Fig. 8 , it can be seen that four of the five compounds showed better agreement between duplicates for all methods when manually integrated, as expected. The fifth compound, isoimperatorin, showed similar results between summation and manual integration across the three methods. This indicates that there is a significant amount of residual background in four of the components which can be excluded with manual integration. In these data, the concentrations of analytes were low in many cases (described in further sections) which led to small signal-to-background ratios. This amplified the impact of any residual background in the resolved components. For higher signal-to-background ratios, the residual background would have a lesser effect on peak area calculations. While manual integration is considerably more tedious, the results are superior in terms of quantification for this data.

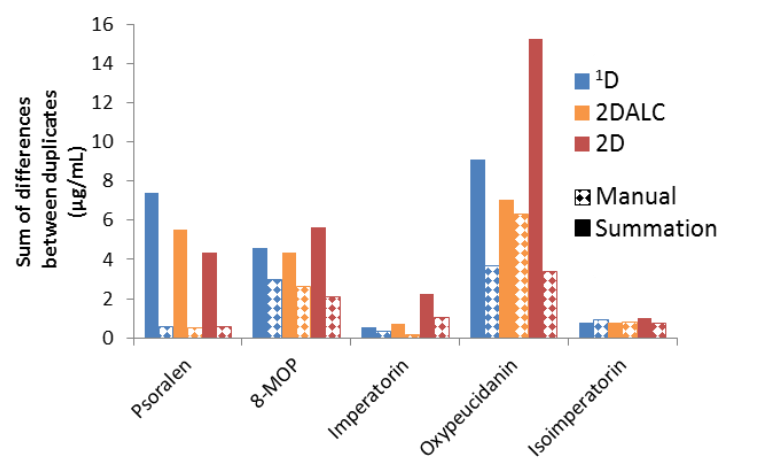

Figure 8. Comparison of MCR-ALS approaches applied. The differences in estimated concentration between 26 duplicate pairs of parsley samples were summed and are compared across MCR-ALS methods and integration 
methods. The patterned bars show the results when the peaks are manually integrated while the solid bars show when the resolved chromatograms are integrated over the entire component.

\subsection{Experimental Data -- Comparison of Curve Resolution Strategies}

In addition to manual versus summation approaches, it is important to understand the performance of the three methods tested $-{ }^{1} D, 2 D$, and $2 D A L C-$ for the experimental data analyzed. Figure 8 compares the agreement between duplicates for the three methods tested. MCR-ALS fit information (number of components, fit errors, etc.) is listed in supplemental tables S.2, S.3, and S.4. When comparing the ${ }^{1} \mathrm{D}$ and $2 \mathrm{D}$ data analysis using manual integration, the results are similar, with the 2D analysis having better agreement between the duplicates for 8-MOP and isoimperatorin, while imperatorin agrees better with ${ }^{1} \mathrm{D}$. However, note that these differences are not very large. Overall, ${ }^{1} \mathrm{D}$ and $2 \mathrm{D}$ are similar due to the ability of manual integration to exclude interferents and background. Overall, the agreement between duplicates for the simple summation approach is worse than for manual integration due to the residual background in all methods. For most of the compounds the ${ }^{1} \mathrm{D}$ analysis has better agreement than the 2D analysis, with the exception of psoralen, which eluted early and had more interferents that may not have been completely resolved with MCR-ALS. Interestingly, when manual integration was applied, 2DALC had the best agreement only for imperatorin; however, for the summation approach, 2DALC performed the best or was equal to the best method for all but psoralen. For summation, residual background dominates the errors in quantification. The 2DALC approach minimizes this residual background more than the other methods, leading to superior quantitative results. When manual integration is performed, residual background is mostly eliminated from peak area calculations for all methods, leading to improved quantification across all methods. The remaining errors result from deviations from the true peak area. In this case, the $2 \mathrm{D}$ analysis was deemed to be superior for this data because the agreement between duplicates was best or tied for best among the three implementations of MCR-ALS for all but imperatorin. 

utilized the ${ }^{1} \mathrm{D}$ data provides superior results due to the fact that the signal-to-background ratio is higher in the ${ }^{1} D$ chromatograms. This is, however, limited to cases in which there are no interfering compounds, meaning that an interferent would have the same spectrum in the same resolved component as the target compound. In the presence of interfering compounds the additional chromatographic resolution provided by $L C \times L C$ is very valuable.

\subsection{Vegetable Results (from 2D with Manual Integration)}

Concentrations for the furanocoumarins in all three vegetable types were determined using the 2D chromatograms with manual integration, which was found to be the most precise method tested here. The results are shown graphically in Fig. 9 and numerically in the Supplemental Information in Tables S.5, S.6, and S.7.. Only the ten compounds detected are shown in this figure. Four compounds trioxsalen, phellopterin, epoxybergamottin, and bergamottin - were not detected in any vegetable type in this study. It is interesting to note the wide range of concentrations for many of the compounds even within the same vegetable type. Overall, the parsley samples had the highest concentrations of furanocoumarins, followed by parsnips, and then celery, on a per gram basis, but since the typical serving of parsley is much smaller than parsnips or celery, this does not reflect an actual dietary load. 

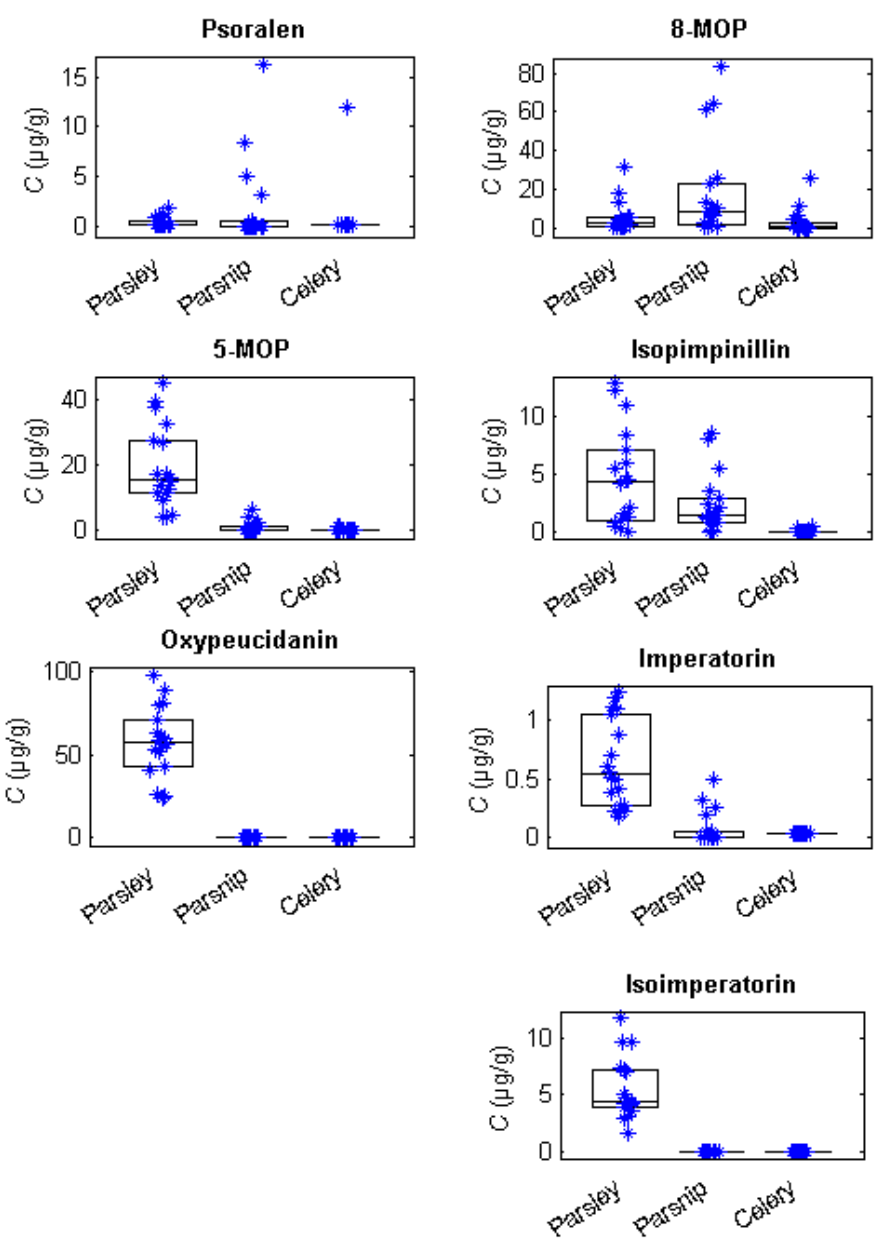
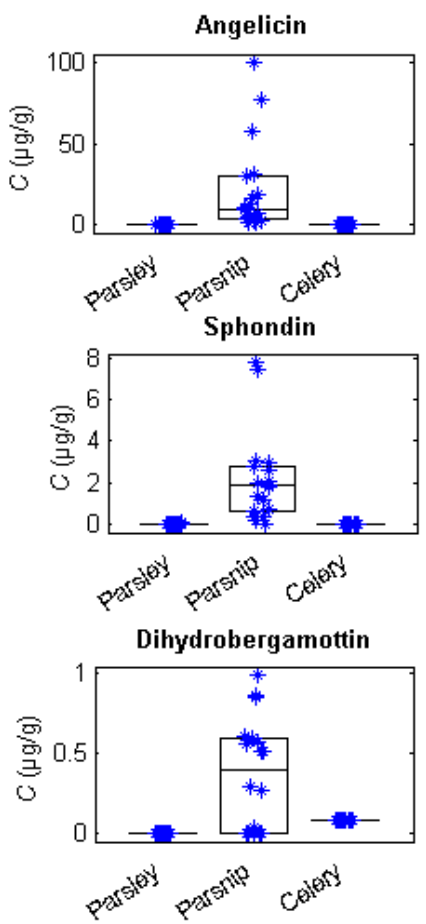

Figure 9. Concentration ( $C, \mu \mathrm{g} / \mathrm{g}$ fresh weight) of detected furanocoumarins in the three vegetables as calculated with manual integration of the $2 \mathrm{D}$ chromatogram. The box represents the median and the $1^{\text {st }}$ and $3^{\text {rd }}$ quartiles. Each point represents the average concentration in each sample $(n=2)$. Only compounds which were found to be present in at least one vegetable type are shown.

\section{Conclusions}

When quantitative analysis is performed it is important to consider the impact of all steps of the analysis on the quality of the results. This is especially true when chemometric techniques are used on the data. Here, several strategies for MCR-ALS analysis of LCXLC data were compared, along with the subsequent integration methods. First, the performance of c2DALC was found, through analysis of 
simulated data, to be greatly degraded by differences between the spectra obtained from the ${ }^{1} D$ and ${ }^{2} D$ detectors, which we believe are caused by the difference in mobile phase compositions. While not useable for these data, the c2DALC approach could possibly be used in cases where spectral shapes do not vary between detectors. In most cases 2DALC performed similarly to resolving the ${ }^{1} \mathrm{D}$ chromatogram by itself. Even though spectral shifts were present, the two-step strategy of 2DALC, rather than the onestep of c2DALC, allowed for the ${ }^{2} D$ spectra to be further optimized for the resolution of the ${ }^{1} D$ chromatogram. In cases where target analytes are overlapped with interferents having similar spectra MCR-ALS is unable to resolve the target analyte from these interferents by itself, and additional chromatographic resolution is required. An example of this was observed in the first retention time window analyzed; interferents with spectra identical to psoralen were present. In the present analysis the interferent was sufficiently resolved chromatographically, allowing for manual integration to isolate the target analyte peak. In cases where the spectrally similar interferent is chromatographically overlapped with the target analyte, or a summation approach to integration is desired, the additional chromatographic resolution achieved with LC $x$ LC is indispensable.

As expected, manual integration was superior to summation approaches; however, this approach is time consuming for studies involving many samples and for $2 \mathrm{D}$ chromatograms in which several ${ }^{2} D$ peaks must be integrated for a single ${ }^{1} D$ peak. When summation was used, the ${ }^{1} D$ chromatogram gave better agreement between replicates, but caution must be used so that interfering peaks are not included in the component window being summed. It is possible that the resolved chromatograms could be submitted to peak detection for a more automated method of excluding residual background [26]. Depending on the performance of the peak detection algorithm, results from this approach may approach those obtained from manual integration. This would facilitate the use of the $2 \mathrm{D}$ chromatographic data to obtain superior quantitative results. 
Finally, 14 furanocoumarin compounds were targeted for quantification in three vegetable types. Only 10 of the 14 compounds were detected in this study and were detected at mostly low levels $(<5$ $\mu \mathrm{g} / \mathrm{g}$ in many cases). A large variation in the estimated concentration of analytes was found even within a single vegetable type. This variation may arise from characteristics of the vegetables such as freshness, exact plant species, geographical location, etc.; however, an analysis of these possible correlations was not performed here.

\section{Acknowledgements}

Funding for SCR and DWC was provided by National Science Foundation (NSF) grants CHE1213561 and CHE-1507332. DWC was also supported by a VCU Altria Graduate Fellowship. All instrumentation and columns used in this work were provided by Agilent Technologies. MB and DS were supported by a NSF grant CHE-1213244. Furanocoumarin standards and vegetable samples were provided by Dr. Sabrina Trudo of the University of Arkansas.

\section{References}

[1] A. Dugrand, A. Olry, T. Duval, A. Hehn, Y. Froelicher, F. Bourgaud, Coumarin and furanocoumarin quantitation in citrus peel via ultraperformance liquid chromatography coupled with mass spectrometry (UPLC-MS)., J. Agric. Food Chem. 61 (2013) 10677-84. doi:10.1021/jf402763t.

[2] L. Guo, Y. Yamazoe, Inhibition of cytochrome P450 by furanocoumarins in grapefruit juice and herbal medicines, 25 (2004) 129-136.

[3] H. Canada, H. Products, F. Branch, Furanocoumarins in Celery and Parsnips: Method and Multiyear Canadian Survey, (2001) 1135-1143.

[4] A.-Y. Kang, L.R. Young, C. Dingfelder, S. Peterson, Effects of furanocoumarins from apiaceous vegetables on the catalytic activity of recombinant human cytochrome P-450 1A2., Protein J. 30 (2011) 447-56. doi:10.1007/s10930-011-9350-0.

[5] Psoriasis, Mayo Clin. (2015). http://www.mayoclinic.org/diseasesconditions/psoriasis/basics/treatment/con-20030838 (accessed August 12, 2015).

[6] M.A. Pathak, T.B. Fitzpatrick, The evolution of photochemotherapy with psoralens and UVA (PUVA): 2000 BC to 1992 AD, J. Photochem. Photobiol. B Biol. 14 (1992) 3-22. doi:10.1016/10111344(92)85080-E. 
[7] F. Cacciola, S. Farnetti, P. Dugo, P.J. Marriott, L. Mondello, Comprehensive Two-dimensional Liquid Chromatography for Polyphenol Analysis in Foodstuffs, J. Sep. Sci. In press (2016) 1-58. doi:10.1002/jssc.201600704.This.

[8] J.M. Amigo, T. Skov, R. Bro, ChroMATHography: solving chromatographic issues with mathematical models and intuitive graphics., Chem. Rev. 110 (2010) 4582-605. doi:10.1021/cr900394n.

[9] S.C. Rutan, A. de Juan, R. Tauler, Introduction to Multivariate Curve Resolution, in: S.D. Brown, R. Tauler, B. Walczak (Eds.), Compr. Chemom., Elsevier, 2009: pp. 249-259. doi:10.1016/B978044452701-1.00046-6.

[10] R. Tauler, Multivariate curve resolution applied to second order data, Chemom. Intell. Lab. Syst. 30 (1995) 133-146. doi:10.1016/0169-7439(95)00047-X.

[11] D.W. Cook, S.C. Rutan, D.R. Stoll, P.W. Carr, Two dimensional assisted liquid chromatography - a chemometric approach to improve accuracy and precision of quantitation in liquid chromatography using 2D separation, dual detectors, and multivariate curve resolution, Anal. Chim. Acta. 859 (2014) 87-95. doi:10.1016/j.aca.2014.12.009.

[12] D.R. Stoll, X. Li, X. Wang, P.W. Carr, S.E.G. Porter, S.C. Rutan, Fast, comprehensive twodimensional liquid chromatography., J. Chromatogr. A. 1168 (2007) 3-43. doi:10.1016/j.chroma.2007.08.054.

[13] P.W. Carr, D.R. Stoll, Two-Dimensional Liquid Chromatography: Principles, Practical Implementation and Applications. Primer., 2015.

[14] I. François, K. Sandra, P. Sandra, Comprehensive liquid chromatography: Fundamental aspects and practical considerations-A review, Anal. Chim. Acta. 641 (2009) 14-31. doi:10.1016/j.aca.2009.03.041.

[15] H.P. Bailey, S.C. Rutan, Chemometric resolution and quantification of four-way data arising from comprehensive 2D-LC-DAD analysis of human urine, Chemom. Intell. Lab. Syst. 106 (2011) 131141. doi:10.1016/j.chemolab.2010.07.008.

[16] H.R. Keller, D.L. Massart, Evolving factor analysis, Chemom. Intell. Lab. Syst. 12 (1992) 209-224. doi:10.1016/0169-7439(92)80002-L.

[17] C. Ruckebusch, L. Blanchet, Multivariate curve resolution: a review of advanced and tailored applications and challenges., Anal. Chim. Acta. 765 (2013) 28-36. doi:10.1016/j.aca.2012.12.028.

[18] R. Allen, S. Rutan, Alignment and quantification of peaks using parallel factor analysis for comprehensive two-dimensional liquid chromatography-diode array detector data sets, Anal. Chim. Acta. 723 (2012) 7-17. doi:10.1016/j.aca.2012.02.019.

[19] R. Tauler, M. Maeder, A. de Juan, Multiset Data Analysis : Extended Multivariate Curve Resolution, in: S.D. Brown, R. Tauler, B. Walczak (Eds.), Compr. Chemom., Elsevier, 2009: pp. 473-505. doi:10.1016/B978-044452701-1.00055-7.

[20] A. de Juan, Y. Vander Heyden, R. Tauler, D.L. Massart, Assessment of new constraints applied to the alternating least squares method, Anal. Chim. Acta. 346 (1997) 307-318. doi:10.1016/S00032670(97)90069-6.

[21] P. Cutler, P.J. Gemperline, A. de Juan, Experimental monitoring and data analysis tools for protein folding: study of steady-state evolution and modeling of kinetic transients by multitechnique and multiexperiment data fusion., Anal. Chim. Acta. 632 (2009) 52-62. doi:10.1016/j.aca.2008.10.052. 
[22] D.R. Stoll, E.S. Talus, D.C. Harmes, K. Zhang, Evaluation of detection sensitivity in comprehensive two-dimensional liquid chromatography separations of an active pharmaceutical ingredient and its degradants, Anal. Bioanal. Chem. (2014) 13-15. doi:10.1007/s00216-014-8036-9.

[23] P.H.C. Eilers, A Perfect Smoother, Anal. Chem. 75 (2003) 3631-3636. doi:10.1021/ac034173t.

[24] E. Bezemer, S.C.S. Rutan, Analysis of three- and four-way data using multivariate curve resolution-alternating least squares with global multi-way kinetic fitting, Chemom. Intell. Lab. Syst. 81 (2006) 82-93. doi:10.1016/j.chemolab.2005.10.005.

[25] C. Reichardt, Solvent Effects on the Absorption Spectra of Organic Compounds, in: Solvents Solvent Eff. Org. Chem., 3rd ed., Wiley-VCH, Weinheim, Germany, 2003: pp. 329-388.

[26] B.J. Place, M.J. Morris, M.M. Phillips, L.C. Sander, C.A. Rimmer, Evaluation of the impact of peak description on the quantitative capabilities of comprehensive two-dimensional liquid chromatography., J. Chromatogr. A. 1368 (2014) 107-15. doi:10.1016/j.chroma.2014.09.060. 
Graphical Abstract

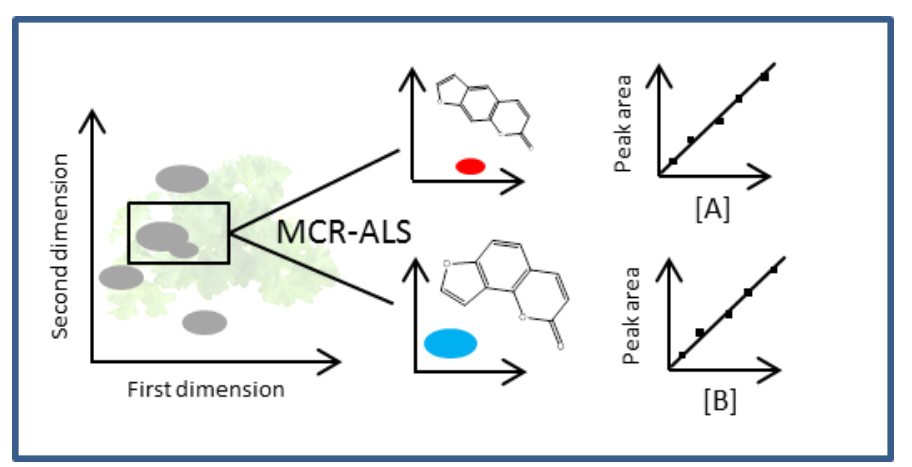

18

19

20

21

22

23

24

25

26

27

28

29

30

31

32

33

34

35

36

37

38

39

40

41

42

43

44

45

46

47

48

49

50

51

52

53

54

55

56

57

58

59

60

61

62

63

64

65 